\title{
Index Volume 3
}

(Numbers followed by asterisk refer to Society Abstracts)

Acetoacetate 90*

Acid-base balance $485^{*}$

Acid base homeostasis $380 *$

Acidosis 160, 364*, 398, 485*

- respiratory $350 *$

Acid phosphatase 516*

Active transport 105

ADAM, P. 380*

ADAms, H.R. 189

Addison's disease 359*

Adels, B. R. 495*

Adenine 356*

Adenosine triphosphatase 105

Adenyl cyclase $349 *$

Adolescence 538

Adolescent 348*

Adrenal gland 373*

- malignancy $374 *$

AeberHARd, E. 590

Agammaglobulinemia 488*

Agathopoulos, A. 92*

Aharon, A.S. 354*

Airway resistance 128

Alberto, R. 491*

D'AlBorA, J. B. 545

Albumin 90*, 366*, 376*

Aldosterone $511^{*}$

Alepa, F.P. 372*

ALFORD, C. A. $498 *$

Allergy 491*

Alper, G. A. 501*

AlPeRs, D.H. 487*

$\alpha_{1}$-Antitrypsin, deficiency $373 *$

Amino acid, clearance 95*

Amino acids 113

Amino acid urea $95^{*}$

Aminoglutethimide 373*

Aminoimidazolecarboxamide 378*

Ammonia 364*

Ammonium chloride $364 *$

Amniotic fluid 113

Amylo-1,4 glucosidase 368*

AnAst, C. 374*

ANders, P.W. 81*, 287

ANDERSON, J. 356*

Ando, T. 269

Androgen 378*

- measurement 373*

Anemia 320, 338

- aplastic 360*

- iron deficiency $366^{*}$

- sickle cell $378^{*}$

Antibody

- response $372 *$

- secretory $359 *$

AnTonovych, T.T 545

Aorta 481*

Apnea 381*

ARIAS, I. M. 5, 171, 352*

Arrhythmia 210
Arthritis, rheumatoid $370^{*}$

Arylsulfatase A 359*

L-Asparaginase 492*

Asphyxia 228, 357*

Assali, N. S. 398

Astruc, M. 93*

August, G. S. 378*

AuLD, P.A. M. 255

AURICCHIO, S. 313

Auriculo-osteodysplasia 370*

Austen, K. F. 373*

Autoimmunity 233, 372*

Autotransplantation 34

AVERY, M.E. 484*, 505*

Azathioprine 363*

BACHMANN, P. 95*

BAKER, L. 368*

BAKKEN, A.F. 205

BALdini, M. 377*

BALFOUR, H. H. 351 *

BALIAH, T. 362*, 597

Barbiturate 355*

BARNESS, L. A. 217

BARNETT, H.L. 363*

BART, R. 361*

BARTSOCAs, C.S. $487 *$

Bartter's syndrome 373*

Basal metabolism 41

BAsinger, G.M. 525

Battaglia, F. C. 60

BAublis, J.V. 358*

Bautista, A.T. 358*

Bavela-StravaKakis, K. 448

BEATTY, C.H. 525

BECK, M.R. 357*

Behavior 199, 275, 305

- child $346 *$

- maternal 346*

- parental 347*

BEHRMAN, R.E. 380*

BELAY, M. 89*

Bellanti, J.A. $372 *, 376 *$

Benke, P.J. 356*, 562

BERAN, A.V. 351*

Berger, D. 380*

BERGMAN, R. A. 484*

BERKOVICH, S. 360*

Bessman, S.P. 366*

Brasucci, A. 371*, 488*

BicARBonAte $364 *$

Bicarbonate threshold 140

Bile 171, 353*

Bile acid $96^{*}$

Biliary atresia $355^{*}$

Bilirubin $90^{*}, 171,205,352 *$, $376 *, 380 *, 448,507 *$

Bissiri, G. R. 348*

BissonnetTe, J. M. 380*

BIXBY, E. M. 487*

BLEGHNER, J.N. 377*
Blizzard, R. M. 538, 579

Blood groups $370 *$

Blood glucose 579

BLOOM, G. E. 369*

Blumenfeld, O.O. 5

BLUNT, M.H. 189

BoceK, R. M. 525

BoDA, D. 89*

Body composition 66

BogGs, D.E. 356*

BOLEY, S. J. 353*

Bongrovanni, A.M. 373*

BOONYAPRAKOB, U. 350*

Bossi, E. 366*

BOWEs, W. 60

BOWMAN, H. 369*

BOWMAN, J. M. 375*

BOWMAN, R. L. 509*

Boyd, E. M. 189

Bradykinin 571

Brain 66, 77, 135, 357*, 358*, $362 *, 366 *, 407,441$

- protein synthesis $357^{*}$

Brain cells 181

BRAsel, J.A. 367*

BRAunstern, H. 358*

BraunWald, E. 481*

BRETTSCHNEIDER, L. 364*

BROWN, D. M. 374*

Bunyaviroch, E. 380*

BurgeoIs, C. H., Jr. 357*

Butler, A. M. 475

BuTTON, L.N. 515*

Calcagno, P. L. 545

Calcitonin $374 *$

Calcium 94*, 95*, 160, 374*

- determination $93 *$

- dietary $91 *$

Caloric deprivation 320, 338

Canale, V.C. $366 *$

Cancer 389, 492*

Candidiasis 502*

Canent, R.V. 350*

CANN, H.M. 369*

CANTZ, B. E. $372 *, 376 *$

Capillary permeability $90 *$

CAPP, M.P. 350*

Carbamyl phosphate synthetase 358*

Carbon monoxide 380*

Cardiac shunt 149

Gardiopulmonary function $349 *$

Garver, M.J. 135

Gassady, G. 498*

Gatecholamines 413

Cell culture 217

Cellular multiplication 66

Central nervous system 181, 498*

Cerebral sclerosis 359*

Cerebro-oculo-renal syndrome 140 
Cerebrospinal fluid 354*

Ceruloplasmin 96*

Chain elongation 590

Chamove, A.S. 305

Ghase, H.P. 347*

Cimeer, D. B. 66, 77, 433, 579

Gherry, R. B. 485*

Child care 199

Ghilgren, R.A. 502*

Cholestasis $96 *$

Cholesterol 93*, 373*

Cholesterol synthesis 597

Choline 367*

Chondroitin sulfate $91 *$

Chown, B. 375*

Chromosomes 96*, 365*, 368*, $369 *, 489 *$

Chung, A. C. $481 *$

Citric acid cycle 217

Glark, L. C., Jr. 351*

GLARK, S. 356*

Cocaine 481*

Gohen, F. 508*

GoHen, M.I. 5, 353*

Colombo, J.P. 95*

Comer, D.S. 357*

Communication 199

Complement 362*, 363*, 501*, 504*

Congenital adrenal hyperplasia $511 *$

Congenital defects 389

Congenital heart disease 210,515*

Constantsas, N. 92*

GonTe, F. 483*

Conway, M.J. 121

COOper, C. 349*, 481*

GopenHAVER, J.H. 135

Copper $96 *$

- metabolism 377*

Cordano, A. 579

Cornfeld, D. $372 *$

Costin, G. 557

CotTER, J.R. 377*

CoWGer, M. L. 376*

Cox, R. 370*, 486*

Cox, S. 371*

Crastes de Paulet,. A 93*

Crastes de Paulet, P. 93*

Creatinine excretion 579

Crigler-Najjar syndrome 490*

Cryoglobulins 363*

CSER, A. 89*

CSERnay, L. 89*

Curse, Ondine's $351 *$

Gurtius, H. C. $91 *, 287$

Gycloheximide 367*

Cystathionase $92 *$

Cystathionine

- synthetase 92*

Cystic fibrosis of the pancreas $347 *, 571$

Cystine 364*

Cystinosis $92 *, 364 *$

Cytology 228

Cytomegalovirus $361 *$

Dahlenburg, G.W. 149

DAILY, W.J.R. 244
Dallman, P.R. 377*

DAncis, J. $486^{*}$

Davies, R. 380*

Davis, N.C. 363 *

Deasy, P.F. 545

Deafness 545

Degeneration, fatty $357 *$

Dehlinger, J. 493*

DELEMOS, R.A. 505*

Delivoria-Papadopoulos, $\mathrm{M}$. 376*

DENNY, F.W. 464

DENT, P.B. 374*

DERrICK, C.W. 362*

Development

- behavioral 347*

- biochemical 365*, 371*, 376*

- cellular 366*, 367*

- growth $369 *$

- pharmacological $351 *$

- physiological $349 *, 374 *, 379 *$

Developmental biochemistry 5 , $27,171,313,407,481 *, 488 *$, $512 *, 514 *$

Developmental biology 305

Developmental pharmacology $481 *$

Diabetes mellitus 510*

Diamond, L.K. 459

Diarrhea 96*

Dibutyryl cyclic AMP 367*

Dierks-Ventling, C. 366*

Dillon, H.C. $362 *$

Dilts, P.V., Jr. 398

Di Martino, D. 313

DiMauro, S. 368*

Dimitrov, N.V. 217

2,3-Diphosphoglycerate 376 *

Disaccharide 96*

DJARDJOURAS, E. M. 93*

Doershuk, C.F. 128

Donaldson, M.H. 378*

DonATH, A. 94*, 95*

DONE, A.K. 348*

Douglas, S. 361*

Doxiadis, S. A. 448

Dozy, A.M. 189

DroEse, W. 91*

DRUMMOND, K.N. 362*, 597

DUBilier, L.D. 358*

Dubors, R.S. 353*

Ductus arteriosus 149

Duffy blood type $370 *$

Duncan, B.R. 347*

EBERLEIN, W.R. 373*

ECK, E. 89*

Ectodermal dysplasia 488*

Edelmann, C. M., Jr. 364*

Edema, cerebral 357*

EDWARDS, N.K. 381 *

EGAN, T.J. 516*

EICHEL, B. 275

EldJARN, L. 92*

Embryology 365*

Emphysema 373*

Encephalitis 358*

- Dawson's inclusion 362*

Enzyme activity 525
Enzyme induction 205

Enzymic activity 571

Epilepsy 298

Epinephrine 77, 354*, 367*

EPSTEIN, G.J. 365*

Epstein-Barr virus 361*

Erythroblastosis $375^{*}, 380^{*}$

- fetalis $60,375^{*}, 380^{*}, 508 *$

Erythrocyte 369*, 373*, 374*, $376^{*}, 508 *$

- lipid 355*

- lipids 93*

Erythrocytes 105, 220, 279

Erythrokinetics 338

Erythropoiesis $189,320,338,378 *$

Erythropoietin 338

Esophagus 353*

Euglobulin 233

EvIATAR, L. 359*

Evoked responses 357*

Exchange transfusion 90*

Extracellular water 140

Eye 504*

FALK, G.S. 413

FANCONI, A. 95*

Fanconi's anemia 96*

Fat, dietary $91 *$

Fatty acid oxidation $365^{*}$

Fatty acid synthesis 590

Fatty acids $91^{*}$

FAULK, W.P. 354*, 499*, 510*

Febrile convulsions 298

Ferencz, G. 351 *

Ferguson, T. B. 353*

FERREIRo, J. 379*

Fetal hemoglobin 189

Fetal-like glomeruli 545

Fetus $90^{*}, 113,121,181,313$, $366^{*}, 367 *, 373 *, 375 *, 377 *$ $380 *, 481 *, 484 *, 505 *, 512 *$

Fibroblasts $217,368 *, 532$

FIKRIG, S. M. 360*

FinBeRG, L. 354*

Fine, B.P. $364^{*}$

Fine, R.N. 346*, 365*

FISH, I. 407

FISHER, D. A. 375* 378*

FLETCHER, G. 244

Foam cells 545

FofT, J.W. 498*

Folic acid $356^{*}$

Follicle stimulating hormone $488^{*}, 538$

FOLWELL, J. 374*

FONKALSRUD, E. 505*

Foramen ovale 149

FORD, J.D. 441

ForRISTAL, J. 363*

Fortin-Magana, R. 514*

Francis, V. 346*

Frasier, S.D. 557

FREDRICKSON, D.S. 368*, 532

Freeman, L. M. 353*

Friedman, W. 349*, 481*

Fucalllo, D. A. $362 *$

FuDENBERG, H.H. 354*, 499*, $510^{*}$

Fulginiti, V.A. 360* 
606

Gajdusek, D. C. 495*

Galactokinase 368*

Galactose 279, 441

Galactosemia 279, 368*

$\beta$-Galactosidase 368*, 532

Galactose 1-phosphate 279

Gall, G. 373*

Ganglioneuroma 413

Gangliosides 532

Gangliosidosis 368*

GARELL, D. C. 348*

Gargoylism 19

GARTNER, L.M. 5, 171

Garzon, A. A. 349*

GaTTI, R. 94*, 95*

GatTi, R.A. 488*

GAULL, G.E. 92*

Genetic disease 287, 358*, 359*, $368 *, 369 *, 370 *, 371 *, 373 *$ 376*, 486*, 488*, 490*, 502*, $504 *, 516 *$

Genetics $91 *, 95 *, 96 *, 354 *, 483 *$

Germain, D. 96*

GerRItSEN, T. 269

Gessner, I.H. 349*

Gestation 499*

GHADIMI, H. 355*

GiBBs, C.J., Jr. 495*

Gibson, L. E. 347*

GrTHeNs, J.H. 371 *

Gitlin, D. 371*, 488*

Grtzelmann, R. 279

GLADE, P. 361*

GLasgow, L. A. 361*

$\gamma$-Globulin 371*

Glomerular filtration rate 51

Glomerulonephritis 363*, 513*

- acute 362*

Glucagon 349*

Gluconeogenesis 364*, 366*

Glucose 90*, 121, 160, 366*, $368 *$

- blood 90*

- 6-phosphate dehydrogenase $365 *, 448$

- 3-o-methyl 353*

- Tm 51

- transport 355*

Glucuronidation 171

Glucuronyl transferase 171

Glutamine synthetase $366^{*}$

Glutaminyl proline dipeptidase 313

$\gamma$-Glutamyl transferase 5

Glycine 269, 356*

Glycogen 121, 366*, 525

Glycogenosis $160,368 *$

Glycogen synthetase 525

Glycyl proline dipeptidase 313

Glyoxylate 269

$\mathrm{G}_{\mathrm{M} 1}$ gangliosidosis 532

Gold, E. 361*

Goldman, A. S. 365*, 373*

Goldstern, S. 349*

Gonadotropins 512*, 538

Good, R.A. 374*, 376*, 488*, 502*

Goodman, J. 499*

Goodman, J.R. 363*, 377*
Index Volume 3

Goodman, S. I. 364*, 368*

GOODNER, C.J. 121

GotTlieb, A. 376*

Graham, G. G. 579

Graham, T.P. $350 *$

GRAND, R.J. 367*

Granulomatous disease, chronic $376^{*}$

Graven, S. N. 367*

GRAYSTONE, J.E. 66, 77, 433

GRAZIANI, L.J. 357*

GRECO, J.M. 351*

GREENBERG, R. 413

GreenberG, R.E. 364*, 366*

GREenstein, R.M. 369*

GribBle, T.J. 378*

Grippo, J. 590

Growth 66, 77, 160, 374*, 407, $485 *, 579$

- failure 305

- hormone 77, 375*, 488*, 579

- kidney 51

- retardation 433, 441, 488*

Grumbach, M.M. 483*, 512*

GRUSHKIn, C. M. 365*

Gunn strain rats $352 *$

GutberLeT, R.L. 379*

GUYDA, H.J. 538

GYePES, M.T. 505*

HadDow, J. 358*, 373*

Hair 493*

HAKAMI, N. 507*

Halvorsen, S. 92*

Hambidge, K. M. 364*

HANSON, L.A. $97 *$

Harlow, H.F. 305

HARNED, H.S., Jr. 379*

Harrah, J. 51

HaRrIS, D. J. 369*

Harrison, V.C. 379*

Hartnup disease 487*

HaworTh, J. C. 441

Heald, F. P. 481*

Heart $210,349 *, 350 *, 365^{*}$, $481 *$

- disease $350 *, 360 *$

Hedvalt, G. 485*

HeIm, T. 89*

Helfer, R. 348*

HeLson, L. 374*

Helwig, F. 348*

Hematuria 545

Heme synthesis $378 *$

Hemodialysis $346 *$, $492 *$

Hemoglobin 60, 220, 376*

Hemoglobin C 189

Hemophilus influenza type B 361*

Henry, G. 354*

Heparitin sulfate $91 *$

Hepatitis, neonatal $96^{*}$

Herbst, J. 514*

Herbst, J.J. 27

Herpes-like virus $361 *$

HerRington, R. 379*

Hertz, H. 90*

Hexokinase 368*

HILL, G. 370*

HIRSGHHORN, K. 361*, 369*
Hirshaut, Y. 361*

Histamine $491^{*}$

Histiocytic medullary reticulosis $372 *$

HoBeL, C.J. 375*

Hoch, M. 363*

HoDson, W.A. 379*

Hollermann, C. E. 545*

Holliday, M.A. 51, 364*, 492*

HOLMES, B. 376 *

HolmGREN, J. 97*

Homocystinuria 92*,368*

HoNG, R. 488*, 502*

Hooks, J. 495*

Hormones 488*, 512*

Hormone excretion 557

Hormone injection 557

HorTA-BARBOSA, L. 362*

Houck, J. G. $481^{*}$

HOWELL, R. R. 359*

HsIA, Y.E. 356*

Hsu, L.Y.F. 369*

Huisman, T.H.J. 189

HuLL, D. 228

Human genetics 389

Human growth hormone 557

HuRwitz, R. 514*

HUTZLER, J. 486*

HUXTABLE, R.F. 351*

Hyaline membrane disease 11

Hydrochlorothiazide 94*

Hydrocortisone 27

Hyperaldosteronism $373 *$

Hyperammonemia $358 *$

Hyperbaric conditions 398

Hypercapnea 485*

Hypercapnia 398

Hypercholesterolemia 597

Hyperglycemia $121,380 *$

Hyperglycinemia $92 *, 269$

- nonketotic $356^{*}$

Hyperlipidemia 93*

Hyperlysinemia 486*

Hyperoxia $351^{*}$

Hyperphosphatasia $358^{*}$

Hyperproteinemia 362*, 597

Hypersensitivity 491 *

Hypoglycemia 66, 121, 354*, 375*

Hypomagnesemic tetany $95^{*}$

Hypophysectomy 433

Hypopituitary dwarf 557

Hypoventilation, alveolar 351 *

Hypoxanthine-guanine phosphoribosyl transferase 356*

Hypoxia 89*, 351*

$\operatorname{Ig} \mathrm{A} 504 *$

IgM 498*

IGO, R.P. $507 *$

IмACH, D. 490*

Immunodeficiency 370 *

Immunoglobulins $34,233,354 *$, $359 *, 362 *, 363 *, 371 *, 372 *$ $375 *$

Immunological deficiency disease 504*

Immunology $361 *, 483 *, 495 *$, 497*, 499*, 501*, 502*, 504* $508 *, 510 *, 513 *$ 
Immunoprotein

- IgG antibody $97 *$

Immunoproteins $371^{*}$

Inanition 121, 433

Infantile marasmus 320, 338

Infants $425,448,579$

Infection 233

Infections, congenital $372 *$

Inflammatory response $497^{*}$

Insulin 66, 77, 121, 375*, 380*, $510 *, 579$

Interferon $361^{*}$

Intestinal 487*

Intestine 5, 27, 96*, 313, 353*, 514*

Intrauterine transfusion 60

Invertase 27

IRIAS, J.J. 364*

Iron metabolism 338, 377*

JACoBowitz, D. 481 *

JacoBson, C.B. 368*, 532

JARAI, I. 41

JARMAKANI, M. M. 350*

JARRAH, A. 51

Jaundice $352 *, 448$

- obstructive $353 *$

JEAN, R. 93*

JEANnet, M. 34

Jejunum $353 *$

Jesse, M.J. 360*

JODAL, U. 97*

Johanson, A.J. 538

JoHnston, R. B., Jr. 501*

JOWSEY, J. 374*

JUNG, A. L. 348*

KABACK, M.M. 359*

KaITA, H. 375

Kallikrein 571

Kaplan, S.A. 375*, 557

KAPLAN, S.L. 512*

KARAKLIS, A. 448

Karlson, K. E. 349*

KARON, M. 490*

Karzon, D.T. 359*

KatHAN, R. 413

KatURICH, N. 351*

Kaufman, D. B. 363*

KAY, H.E. M. 488*

KAZEMI, H. 349*

KeKete, M. 41

KELSCH, R. C. 160

KeNDALL, N. 380*

KenNeLL, J.H. 346*

Keratitis 504*

Keratosulfate 19

KERR, G.R. 305

Keschamras, N. 357*

Ketoacidosis $356^{*}$

Ketosis 354*

KIDD, B. S. L. 379*

Kidney 5, 51, 140, 346*, 362*, $363 *, 364 *, 513 *$

- disease $363 *$

- function $94 *, 364 *$

- transplantation 364*, 365* $492 *, 504 *$

KIESEL, J.L. 358*
KIM, B. $377 *$

KIMBERLING, W.J. 370*

KrNG, K. 380*

Kininase 571

KINSINGER, E. C. $367^{*}$

KIRK, R.F.H. 358*

KIRK, V. 485*

KIRKMAN, H. N. 358*

KirschbaUm, T.H. 398

KistenMACHER, M. 368*

KlaUs, M.H. 346*

KLEIN, R. 358*, 373*

KLEMPERER, M.R. 501*

KNELSON, J.H. 505*

KNIKER, W.T. 513*

KNUTSON, S. $380^{*}$

KOFFLER, H. 354*

KOHN, G. 365*

KOLOBOW, T. 509*

Koprto, L. 493*

KORSCH, B. M. 346*

Koras, R.V. 505*

KOULISGHER, N. 363*

KowALYSHYN, T. 373*

KRASILNIKOFF, P.A. 90*

Krauss, A.N. 255

KRAVATH, R. E. 354*

KRETCHMER, N. 514*

KRETSCHMER, K. 34, 370*

KRETSGHMER, R.R. 34, 370*

Kunze, D. 91*

Kupffer cells 19

KURAMOTO, A. 377*

Lactate 160

Lactoferrin $354 *$

LANGER, L. O. 488*

LANZKOWSKY, P. 366*

LARSEN, C.D. 492*

LA Torretta, G. 313

Launiala, K. 96*

Lead poisoning $493 *$

Lecithin 349*, 367*

LEDLEY, R. 489*

LEIKIN, S. 371*

LEQUIRE, V. 379*

Lesch-Nyhan syndrome 355*, 356*

Leucine sensitivity $355^{*}$

Leukemia 378*, 389

Leukocytes $34,376 *$, 491*, 497*

Leukodystrophy, metachromatic 359*

LeVy, H.L. 113

LEWIS, J. P. 189

LEWIS, M. $375^{*}$

LIEBERMAN, E. 365*

LiEBerman, J. 11, 571

LiLlJEQVisT, A.-C. 356*

LIM, H.S. 380*

LINDE, L.M. 210, 505*

LING, S. M. 557

Lipids, erythrocyte $93 *$

Lipoproteins

$-a 93^{*}$

- $\beta$ 93*

LITTENBERG, G.D. 571

LitTLE, J. M. 504*

LITwIN, S. D. $371 *$
Liver 5, 19, 92*, 171

- cirrhosis $96^{*}$

- disease $353 *$

- function 355*

LLEWELLYN, M.A. 379*

LLOYD, J.K. 93*

LoEB, H. 19

LOGAN, L. 363*

LORIDAN, L. 355*

LOVRIEN, E.W. 370*

LOWENTHAI, A. 495*

Lowy, G. $375^{*}$

LuBs, H. 489*

LULENSKI, G. C. 378*

Lung 11, 244, 255, 349*, 350*, $367 *, 379 *, 484 *$

- compliance 425

- conductance 128

- transplantation 349*, 505*

Luteinizing hormone 488*

LuTz, P. 92*

Luzzatti, L. 369*

Lye ingestion $353^{*}$

LYMAN, M. 491*

Lymph node 34

Lymphocytes $370 *$, 371*, 372*, $483 *, 499 *, 502 *$

Lymphocytosis $361 *$

Lymphopenia $372 *$

Lysine-ketoglutarate reductase 486*

Lysosomes $516 *$

MABRy, G. G. $358 *$

Macaca mulatta 305

Macagno, F. 94*, 95*

Magnesium 95*, 374*, 433

MaISELS, M.J. 380*

MAKowsKI, E. L. 60

Malan, A. 379*

Malnutrition 181, 320, 338

Manganese 433

MAngos, J.A. 562

Marasmus 579

MARKEY, S.P. 368*

MARKS, M. I. $362 *, 597$

Marthaler, T. 91*, 287

MARTIN, H.P. 347*

Matsaniotrs, N. 92*

Matrhews, L.W. 128

Maturation 590

MAUER, S.M. 364*

MAY, C.D. 491*

Maybee, D.A. 376*

MAYER, S.K. $358^{*}$

MaCollum, A.T. 199, 347*

MaCormick, J. N. 354*

MaGaughey, H.R. 60

MaIntosh, R. M. 363*

MaLaine, P.N. 362*, 597

MaLean, F.W. $377^{*}$

MaNamara, H. 353*

MCSherRy, N. 562

Measles 358*, 362*, 495*

Mellins, R.B. 351*

Mellman, W.J. 217, 368*

Menkes, J. H. 359*, 590

Menking, M.F. 356*

Mental retardation $487 *$ 
608

Mentzel, H. 485*

Mentzer, W. G. $378 *$

Mereu, T.R. 34

Merrill, E. W. 349*

Meschia, G. 60

MEsel, E. 350*

Mestyán, J. 41

Metabolism

- amino acid $92 *$

- cardiac 365*

Methemoglobinemia 369*

Methemoglobin reductase 369*

Methionine 92*

Methylmalonic acid 217

Methylmalonic acidemia $92 *$

Methylmalonic aciduria 85*, 356*, 368*

MEuwissen, H.T. 502*

MEYER, H. B. P. 244

Michaels, R.H. $372 *$

MiddelKAMP, J. N. 353*

Migeon, C.J. 538

Mikity-Wilson syndrome 255

Miller, A. 189

Miller, M.E. 372*, 497*

MilleR, R.W. 389

Miller, W. 376*

Mitochondria $72 *, 365^{*}, 377 *$

MoE, P. G. 368*

Momma, K. 210, 505*

Montag, P.P. 13

MoORE, E.S. 67*, 364*

MoORE, T.J. 355*

Morquio's disease 19

Morrow, G., 111, 217

Moshang, T., Jr. 373*

Motlagh, F.A. 349*

Mourning $346^{*}$

Mowat, A. P. 352*

Mucopolysaccharide $91 *$

Murdock, A. I. 379*

Murphy, M. L. 374*, 492*

Muscle 358*

Myelination 590

Myelin synthesis 590

Myotonia 358*

NACHMAN, R. 484*

NADAs, A.S. 515*

NADLER, H. L. 516*

NADPH-cytochrome $c$ reductase $352 *$

Nathan, D. G. $378 *, 515 *$

Nathenson, G. 371 *

Nelson, N. M. 380*, 485*

Nephrectomy $363 *$

Nephritis $363 *, 545$

Nephrosis 93* 363*, 513*

Nephrotic syndrome 363 *

Neurochemistry 181

New, M. I. 511*

Newborn $89 *, 90 *, 91 *, 135,171$, 205, 244, 313, 346*, 357*,

$361^{*}, 366^{*}, 367 *, 376^{*}, 377^{*}$, 378*, 441, 497*, 498*

Newborn infant 128, 149

Newborn infants 181

Newcombe, D.S. 378*

Nicolopoulos, D. $92 *$

Index Volume 3

Nordio, S. 94*, 95*

Norepinephrine 413, 481*

Norman, A. 96*

Noyes, W.D. 377*

Nucleic acid, synthesis $375 *$

Nunnery, A. 275

Nurse practitioner $347^{*}$

Nutrition 27, 66, 77, 91*, 305 , $347 *, 492 *$

NyHAN, W.L. 269

Nymph, water 351 *

Obenshain, S. 380*

Obesity 355*

O'Brien, D. 368*

ODELL, G. B. 351 *

ODELL, W.D. 375*, 378*

OETLIKER, O. 140

OGRA, P. L. 359*

OKuMa, M. 377*

OlIVER, T.K., Jr. 379*

Oliver, W.J. 160

Oogenesis $365^{*}$

$0, p^{\prime}$ DDD $374 *$

Operant conditioning 275

OPPENHEIM, J.J. $371^{*}$

Oski, F.A. 105, 368*, 369*, 376*

Osteomalacia 95*

Osteoporosis 94*

OstroW, J.D. 352*

O'Sullivan, P.M. 96*

OTRIDGe, B.W. 377*

Ovary $365 *$

Oxygenation 149, 398

Oxygen consumption 41

Oxyhemoglobin 60

PAGe, L.A. $366 *$

Paltrowitz, I. 361*

Pancreatic insufficiency 562

Pancreatin 562

Paralysis, periodic $358^{*}$

Paramyotonia $358 *$

Parathyroid gland $374 *$

Parent-physician relations 199

PARK, B. H. $376 *$

Parkinson, D. K. 374*

Parotid gland $367^{*}, 562$

Parotitis $372 *$

Pathak, A. 380*

PAYNE, F.E. 358*

Peddle, L.J. 375*

Pediatric care $347 *, 348 *$

- teaching $348 *$

D-Penicillamine $96 *$

Penicillin 360*

Penn, I. 364*

Perakis, A. 448

Perex, D.Y.E. 374*

Perlstein, P. H. 381*

Peyer's patches 354*

Phagocytosis $376 *, 501 *$

Pharmacology $348 *$

Phelan, P.D. 425

Phenylalanine $91^{*}, 287,305$

Phenylketonuria 91*, 287

Phenylpyruvic acid

- $p$-hydroxy $92 *$

Phillppe, N. 96*
Philippon, F. 353*

Phosphatidyl choline 93*

Phosphoenolpyruvate 369*

Phospholipid 93*

Phosphorus 95*

Phosphorylase 525

Phototherapy $352 *$, 380*, 490*, 507*

PIeL, C. $363 *$

Pierce, J. E. 509*

Pierro, M. 313

Pineda, G. 357*

Pituitary 77

Placenta 366, 499

- artificial 509

Plasma concentration 557

Plasma growth hormone 557

Plasma infusion 597

Plasminogen 11

Platelets 377*

Platt, N. 488*

Plumb, N. 346*

Poisoning $348 *$

Poliovirus 359*

Pollak, S. 368*

Pollock, J.M. 375*

Pollock, P. G. 358*

Polt, S.S. 498*

Pomerance, H.H. 488*

Pool, P. 481*

Potassium 105, 358*

Potter, D. 51

PotTer, D.E. 364*, 492*

Prader, A. 95*

Prednisone 363*

Pregnancy 113, 398, 441

Premature infant 105, 255

Prematurity 11, 41, 135, 298, $379 *, 381 *, 485 *$

Prindiville, T. 513*

Propionate 217

Protein 407

- plasma 90*

Protein synthesis $135,366 *, 367 *$

- brain 357*

Proteinuria 362*, 597

Protoporphyrin 378*

Pseudocholinesterase $370 *$

Puescheld, S. M. 493*

Pulmonary artery $351 *$

Pulmonary function 128

Pulmonary resistance 425

PunnetT, H.H. 368*

Pyelonephritis 97*

Pyridoxine 92*

Pyruvate kinase $369 *$

Pyuria 97*

QUIE, P.G. 502*

RADDE, I. G. 374*

Radioimmunoassay 538

Radioulnar synostosis $369 *$

RaIHA, N. 380*

Rarvio, K. 380*

RaIvro, K. O. 90*

RAMES, L. 365*

RampInI, S. 91*, 287

Rassin, D. K. 92* 
Rat brain 590

Rats 562

RAYE, J.R. 379*

Redman, W. 360*

REES, E. 350*

ReEves, M.S. $362 *$

ReHBeRG, M.L. 269

REID, M. MCC. 379*

Renal epithelial cells 228

Renal function $51,364 *$

Renal hemodialysis $365^{*}$

Requin, C. $96 *$

Respiration 128, 255, 350*, 425

Respiratory distress syndrome $11,89 *, 149,244,255,357 *$, $379 *, 485 *$

Retardation

- growth $358^{*}$

- mental 355*, 356*, 358*, 369*

Reticulocytosis 105

Rh 370*

Rhesus muscle 525

Rickets 95*

- vitamin D deficiency, pseudo 95*

- vitamin D-resistant 95*

RobBins, J. B. 354*, 361*

Roberton, N.R.C. 149

RodRIGUES, L. P. 361*

Romshe, C.A. $356 *$

ROPER, C.A. 353*

Rosario, E.S. 360*

Rose Bengal 353*

Rosen, F.S. 370*, 501*

Rosen, S. 34

RosenberG, L.E. $356 *$

Rosenthal, A. 515*

RosenthaI, I. M. 413

Rosenthall, L. 353*

Rossi, E. 94*, 140

Rosso, P. 181

Roy, C.C. 353 *

ROYER, P. 93*

Rubella virus $372 *$

RuBino, A. 313

RUDD, B.T. 373*

RUDDLE, F. 489*

SAdEGHi-Nejad, A. 355*

SAKAI, T. 51, 364*

Salicylate $358^{*}$

Saliva 571

Salivary flow rates 562

SARDHARWALLA, I. B. 96*

SASS-KorTSAK, A. $96 *$

SATRASOOK, S. S. 364*

Schain, R.J. 135, 357*

SchaLrer, J. $370^{*}$

SchrFe, D. 375 *

SGHLEGEL, R. J. 376*

SCHORR, J.B. $37 l^{*}$

SCHROTER, W. $90^{*}$

Schubert, W. K. 354*

SCHULKIND, M.L. 504*

SchWARTZ, A. $490^{*}$

SchWARTZ, A.H. 199

SGHWARTZ, H.C. 378*

Schwartz, M. 492*

SchWARTZ, R. 380*

ScotT, G.R. 356*
ScotT, R. 275

Scully, K. 356*

Seaman, M.P. $511^{*}$

SEEDS, A.E. 380*

Seizures 298

Senror, B. 355*

Sensitivity, cutaneous 502*

Septal defect 349*

- ventricular 350*

Serine 269, 356*

Serum luteinizing hormone 538

Sever, J. L. 362*

Sexual development 538

Shacklady, M. M. 93*

Shannon, D. C. 349*

Shasby, S. $378 *$

ShePard, T. H. 512*

SheRMETA, D.W. 505*

SHIH, V.E. $487 *$

Shimizu, G. S. $375 *$

SHWAGHMan, H. 493*

Sickle cells 220

SYFRI, E. G. 504*

SILVER, H.K. 347*

SiLVERBERG, M. 353*

Simmons, J. 492*

SingSEN, B. $490^{*}$

Stsson, T.R. C. 380 *

Skin $84^{*}, 362 *, 368^{*}$

SLOAN, H. R. 368*, 532

SLOOFE, J.P. 233

SLYTER, H. $346^{*}$

SMITH, C. A. 485*

Smith, N.J. 320, 338, 507*

SMITH, P. C. 244

SOBEL, B. 349*

Sodium 105

Sol-gel formation 220

SoLTÉsz, G. 41

Sotos, J.F. 356*

SOYKA, L.F. 352*

SPACH, M.S. 350*

SPEAR, G.S. 484*

Specific dynamic action 41

Sphingomyelin $93 *$

SPITZER, R.E. 363*, 504*

SPRUNT, K. $360^{*}$

STAHLMAN, M.T. 379*, 485*

STARR, J. 361*

Starvation 89*

StarzL, T.E. 364*

Steatorrhea 96

STEENDIJK, R. 95*

STEINER, M. 377*

Stekel, A. 320, 338

Steroidogenesis $373 *$

Steroids 353*, 359*, 365*

STEVENS, G. 505*

Stinson, D. 350*

Stitzel, A.E. 504*

Stoelinga, G. B. A. 233

STOKKe, O. $92 *$

STOLley, H. 91*

STRANDVIK, B. 96*

StRauss, J. $351^{*}$

STRAVRAKAKIS, D. 448

STREBel, L. 351 *

Streptococcal infection $360 *, 362 *$

Structural genes 189
Sturman, J. 92*

Subacute sclerosing panencephalitis $495^{*}$

Submaxillary glands 562

Succinate 217

Succinylcholine $370^{*}$

Succinyl-CoA $92 *$

SUNDELL, H. 379*

Sunshine, P. 27

Surface tension $349 *$

Surfactant $505^{*}$

Sutherland, I. M. 381*

SuVATte, V. $371 *$

SWYER, P.R. 379*

Talamo, R. C. $373 *$

TALBOT, N.B. 47

TAILAL, L. 492*

TAN, C. $492 *$

TAN, K. 228

Tangier disease $93 *$

TANIGUChI, A. $490^{*}$

Tautomerase $92 *$

TAYLOR, G. 244

TAYLOR, P. 350*

TAYSI, K. 368*

Teller, W.M. 91 *

Temperature, environmental 89*

TENG, C. $356^{*}$

Teramo, K. 90*, 380*

Tetany, hypomagnesemic 95*

ThaxeR, M. M. 355*

Thermogenesis 41

ThIER, S. O. 487*

Thrombocytopenia $377 *$

Thurston, J.H. 358*

Thymic dysplasia 34

Thymus 370*, 371*, 372*

Thyroid 367*, 375*

Thyroid stimulating hormone 488*

Thyrotropin 378*

Tiva, L. U. 545

Tissue culture $359 *$

Titratable acidity 140

Tomsovic, E. J. 510*

TONDEUR, M. 19

TORRES, R.R. 349*

Tracheostomy 275

Transformation, blast $371^{*}$

Transplantation

- kidney $364 *, 365 *$

- lung 349*

Transport

- calcium $374 *$

- erythrocyte $355^{*}$

- glucose $355^{*}$

- Na, K 373*

Transposition of great vessels $350 *$

Tubular acidosis 140

TULZER, W, 89*

Tumor 413

TuRuNo, G. M. 351*

TURNER, S. 505*

Tyrosine 287

- transaminase $92 *$

UDP-glucuronyl transferase $205,351 *, 352 *$ 
610

UHLENDORF, B.W. 368*, 532

Uric acid 355*, 356*

Uridine diphosphate galactose 279

Urine 228

Uterus 398

Vaccinia-A 360*

VAEUSORN, O. 484*

VAITUKAITIS, J. 373*

VALAES, T. 448

VAllota, E.H. 363*

VAN DEN BERG, B.J. 298

Van Munster, P.J.J. 233

Vena cava 210

Ventilation, artificial 244

VERGEL, Z. M. 364*

Vetrella, M. 313

Virus

- Coxsackie B $360 *$

- disease $361 *$

- measles 358*

- Semliki Forest $361 *$
Index Volume 3

Vitamin $B_{12} 356 *$

Vitamin D $91 *, 95 *$

VogleR, G. 90*

WaISMAN, H. A. 305

WALKNOWSKA, J. 483*

Walravens, P.A. 364*

WANG, A. C. $499 *$

WARSHAW, J. B. 365*

WATANABE, K.S. 357*

Watson, D. 350*

Weaning 27

WEDGWOOD, R.J. 370*

WEITZMAN, E.D. 357*

WENNBERG, R.P. 376*

WERRBACH, J.H. 121

West, C. D. $363^{*}, 504 *$

WHAUN, J.M. 105

WhrTe, J.G. 220

Williams, H. E. 425

WInBERG, J. $97 *$

WiNICK, M. 181, 367*, 407

WinN, H. 34
WrNTERs, R.W. 351*

Wiskott-Aldrich syndrome 377 *

WOLFSDORF, J. 484*

WOLFSON, D.R. 354*

WOLLNER, N. 374*

WONG, R. 413

WoODRUM, D.E. 379*

Woody, N. C. $486 *$

WRIGHT, L. 275

Yakovac, W. C. 372*

YERUSHALMY, J. 298

YoFFEY, J. M. 354*

YounoszaI, M.K. 441

ZaGhMAN, R.D. 367*

ZAPOL, W.M. 509*

ZARKOWSKY, H.S. 369*

Zetterstrom, R. 96*

Zinc 433

ZUEHLKE, S. 346*

Zuelzer, W.W. 508* 